\title{
Influence of Pneumoperitoneum and Trendelenburg Position on Fluid Responsiveness Predicted by Pulse Pressure Variation and Stroke Volume Variation During Anaesthesia for Laparoscopic Gynaecological Surgery
}

\section{TULAY SAHIN, LUTFIYE BASTURK ALTAN}

Kocaeli University Medical Faculty, Department of Anesthesiology and Reanimation, Kocaeli, Turkey

Purpose: The increased intraabdominal pressure and intrathoracic pressure due to pneumoperitoneum negatively affect the cardiovascular system, relatively dynamic parameters may vary due to intraoperative fluid therapy. In our study, we aimed to evaluate how dynamic parameters such as stroke volume variation (SVV) and pulse pressure variation (PPV) are affected during the Trendelenburg position and pneumoperitoneum in patients who will undergo an elective gynaecological laparoscopic surgery and their reliability in assessing response to fluid administration.

Methods: After obtaining approval of the IRB and written informed consent, 45 patients aged between 18-65 years, undergoing elective laparoscopic gynaecological surgeries were included. After the general anaesthesia induction and intubation, dynamic parameters such as SVV and PPV were monitored by pulse contour analysis. Before and after pneumoperitoneum combined with the Trendelenburg position ( $30^{\circ}$ degrees), $5 \mathrm{~mL} \mathrm{~kg}^{-1}$ bolus Ringer's lactate was administered and SVV, PPV were measured for evaluation of the fluid response. HR, MAP, and $\mathrm{Cl}$ were also evaluated.

Results: We observed that PPV and SVV values decreased after fluid loading in the pre and post-pneumoperitoneum period. PPV and SVV values were also low after the Trendelenburg position. Although there are measurable changes in hemodynamic parameters due to insufflation and patient position during laparoscopy, these changes were not reflected in the clinic.

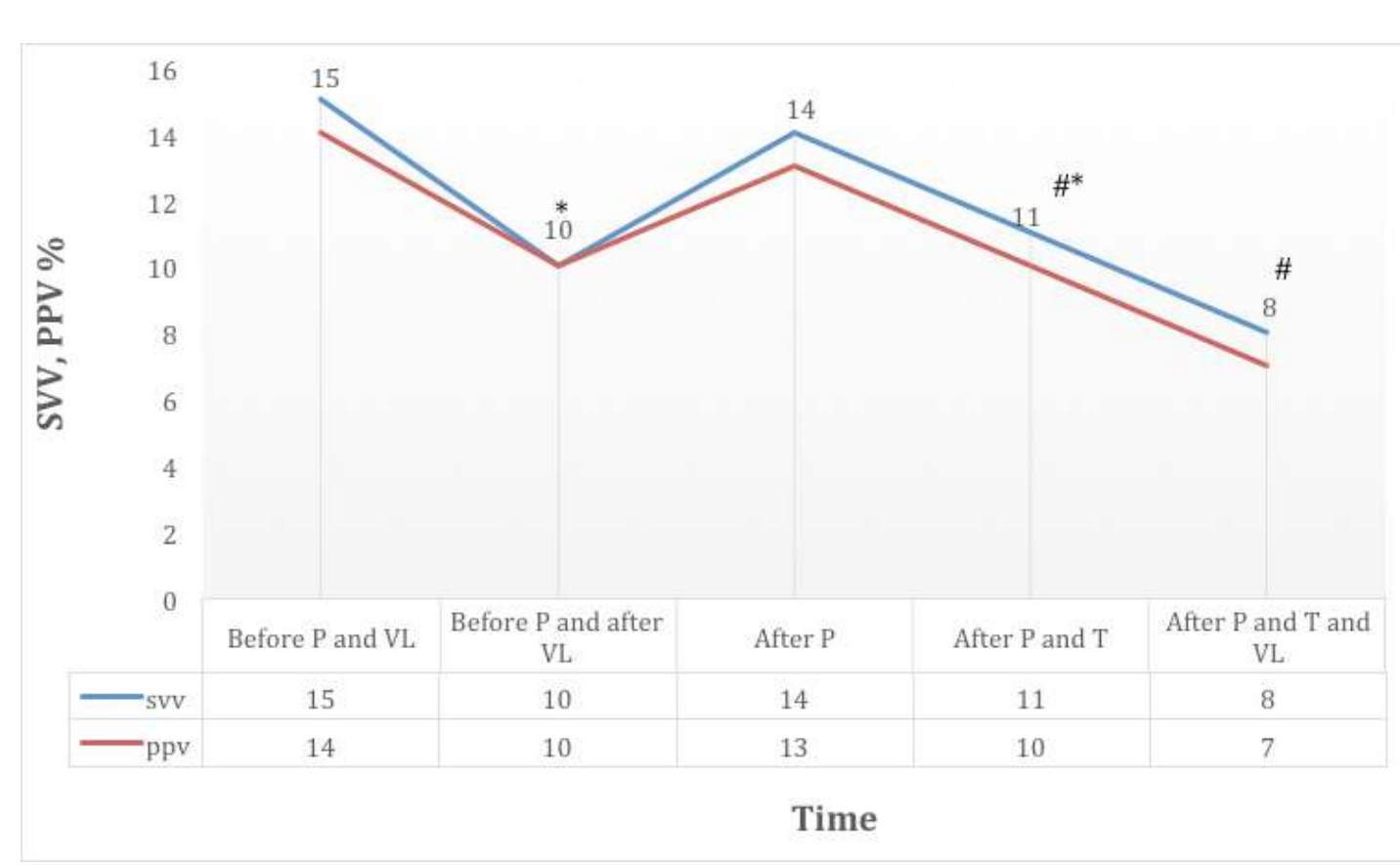

Figure 1. SVV and PPV before and after volume loading, pneumoperitoneum and in the Trendelenburg position $*: p<0.01$ vs. control values.

$\#: p<0,01$ vs. after pneumoperitoneum value.
Conclusions: In this study we observed that pneumoperitoneum did not significantly affect the dynamic parameters in terms of fluid response. SVV and PPV values were reliable in assessing response to fluid administration during the Trendelenburg position and pneumoperitoneum.

Table 1. The patients' demographic data, perioperative characteristics and duration of anesthesia and surgery (Number of patients= 45).

Values are mean \pm SD or number (percentage).

\begin{tabular}{|l|l|}
\hline Age (year) & $32,5 \pm$ \\
\hline Weight (kg) & $65,7 \pm 11,2$ \\
\hline Height (cm) & $162,6 \pm 5,3$ \\
\hline BMI (kg/m²) & $25,4 \pm 5,6$ \\
\hline ASA I/II n (\%) & $40(88) / 5(11)$ \\
\hline Fasting time (hr) & $10(9-10,5)$ \\
\hline Duration of Anesthesia (min) & $100(90-115)$ \\
\hline Duration of Surgery (min) & $115(105-130)$ \\
\hline
\end{tabular}

Table 2. Hemodynamic variables of patients before and after volume loading, pneumoperitoneum and Trendelenburg position

\begin{tabular}{|c|c|c|c|c|c|}
\hline & Before $\mathrm{P}$ and VL & Before $P$ and after $V L$ & After $\mathrm{P}$ & After $\mathrm{P}$ and $\mathrm{T}$ & After $\mathrm{P}+\mathrm{T}+\mathrm{VL}$ \\
\hline $\mathrm{HR}\left(\right.$ beat. $\min ^{-1}$ ) & $83,1 \pm 15,4$ & $82,3 \pm 14,03$ & $82,2 \pm 11,2$ & $80,2 \pm 12,6 \#$ & $77,4 \pm 11,7$ \\
\hline MAP (mmHg) & $76(67,2-87,7)$ & $81(74,2-90,7)^{*}$ & $94(83-104)$ & $101(83,5-110) \beta$ & $98(87,5-109,5)$ \\
\hline $\mathrm{CI}\left(\mathrm{L} / \mathrm{min} / \mathrm{m}^{2}\right)$ & $3,00(2,68-3,46)$ & $2,9(2,45-3,68)$ & $2,83(2,24-3,54)$ & $2,93(2,17-3,67)$ & $3,15(2,33-3,88)$ \\
\hline PPV (\%) & $14(7,5-11,5)$ & $10(12-16)^{*}$ & $13(9,5-14)$ & $10(7-13)$ *\# & $7(6-9) * \#$ \\
\hline SVV (\%) & $15(14-17,5)$ & $10(12-16)^{*}$ & $14(12-18)$ & $11(9-14)$ *\# & $8(7-10,5) * \#$ \\
\hline
\end{tabular}

P: Pneumoperitoneum, VL: volume loading, $\mathrm{T}$ : trendelenburg position, PPV: pulse pressure variability SW: Stroke volüme variability, HR: Heart rate, MAP: Mean arterial pressure $*$ : $<0.01$ vs. control values.

\#: $p<0,01$ vs. after pneumoperitoneum value.

$B: p<0.05$ vs. before trendelenburg position values. 